\title{
The Impact of Personality Traits and Acculturation on the Mental Health of Korean American Adolescents
}

\author{
Minjeong Kim¹, Jyu-Lin Chen ${ }^{2}$, Susan Kools ${ }^{3}$, Sandra Weiss ${ }^{4}$ \\ ${ }^{1}$ School of Nursing, San Diego State University, San Diego, USA \\ ${ }^{2}$ Department of Family Health Care Nursing, University of California, San Francisco, USA \\ ${ }^{3}$ Department of Family, Community and Mental Health Systems, University of Virginia, Charlottesville, USA \\ ${ }^{4}$ Department of Community Health Systems, University of California, San Francisco, USA \\ Email: minjeong.kim2@gmail.com
}

Received 28 June 2016; accepted 22 August 2016; published 25 August 2016

Copyright (C) 2016 by authors and Scientific Research Publishing Inc.

This work is licensed under the Creative Commons Attribution International License (CC BY).

http://creativecommons.org/licenses/by/4.0/

(c) (7) Open Access

\section{Abstract}

Adaptation to a new culture can be highly stressful, especially during challenging developmental stages such as adolescence. The ways in which adolescents adapt to a culture and their resulting well-being may be influenced substantially by their personality traits as well as the degree to which they are aligned with the values of the new culture. Korean Americans are one of the fastest growing immigrant groups in the US, including a burgeoning population of Korean youth. The purpose of this study was to determine whether specific personality traits of Korean American adolescents or their degree of acculturation would be associated with their mental health problems, and whether specific personality traits would moderate the association between acculturation and mental health problems. 138 Korean American adolescents completed a demographic questionnaire, the revised Stephenson Multigroup Acculturation Scale, the NEO Five-Factor Inventory-3, and Achenbach \& Rescorla's Youth Self Report. Hierarchical regression analyses indicated that acculturation played a minimal role in predicting mental health problems, while personality traits were strong predictors. Being more reactive to stress and less emotionally stable (greater "neuroticism") and being less altruistic and cooperative (less "agreeableness") predicted more mental health problems for Korean American adolescents. In addition, the trait of "openness to experience" played a moderating role. For youth who were more open to experience (curious and independent in their judgments), greater alignment with values of the American culture was a protective factor for their mental health. Findings indicate the need for further research to identify types of mental health problems that may be most affected by specific personality traits and the underlying mechanisms responsible for their effects. It will also be important to examine whether personality traits identified in this research influence mental health similarly across cultures and age groups or whether they are unique to Korean American youth.

How to cite this paper: Kim, M., Chen, J.-L., Kools, S., \& Weiss, S. (2016). The Impact of Personality Traits and Acculturation on the Mental Health of Korean American Adolescents. Psychology, 7, 1256-1265.

http://dx.doi.org/10.4236/psych.2016.79128 


\section{Keywords}

\section{Personality, Acculturation, Adolescents, Asian Americans, Mental Health}

\section{Introduction}

Adaptation to a new culture can be highly stressful, especially during challenging developmental stages such as adolescence. However, little is known about how the challenges associated with cultural adaptation may influence an adolescent's mental health.

Korean Americans are one of the fastest growing immigrant groups in the United States (US Census Bureau, 2012). As a result, there has been growing interest in the ways in which acculturation may influence mental health of Korean American youth. In their study of acculturation and adjustment, Cho, Hudley, and Back (2003) found that second-generation Korean American adolescents who were more aligned with the values of the Western culture were more self-reliant and able to face life challenges than their Korean American peers who were less acculturated. In line with these findings, Yeh (2003) reported that greater alignment with the values of Western culture was associated with fewer symptoms of mental health problems for Korean American adolescents. However, Cho \& Bae (2005) reported no relationship between degree of acculturation and the presence of "internalizing" problems such as depression, withdrawal, and anxiety among Korean American adolescents. In contrast, in a study of Korean American youth affiliated with Catholic schools, Lee et al. (2012) found that greater acculturation significantly predicted higher alcohol and drug use. As these studies indicate, there are conflicting findings regarding the association between acculturation and the mental health or adjustment of Korean American adolescents.

The way in which adolescents adapt to a culture may be influenced substantially by their personality traits. Personality traits are the unique and relatively stable patterns of behavior, thought and emotion that distinguish different people. Although a variety of traits have been studied, the five-factor model of personality has been widely examined in research (McCrae \& Costa, 2008; Widiger \& Costa, 2012). This model proposes 5 traits as core dimensions of personality that are present in all individuals to a greater or lesser extent. These include neuroticism (emotional instability, distress in response to stressors), extraversion (sociability, energy, positive emotionality), openness to experience (appreciation of personal emotion, curiosity, independent judgment), agreeableness (trustfulness, altruism, cooperativeness), and conscientiousness (goal directed, self-control, organization). Research has shown compelling links between personality traits and a wide variety of mental disorders (Krueger \& Eaton, 2010), including outcomes of a meta-analysis of 33 studies (Malouff, Thorsteinsson, \& Schutte, 2005). Since early seminal research that examined personality traits in adolescents (Krueger, 1999; Krueger, Caspi, Moffitt, Silva, \& McGee, 1996), studies have continued to indicate that personality traits are strongly associated with mental health outcomes for youth (Cyders, Flory, Rainer, \& Smith, 2009; Hopwood \& Grilo, 2010; Moore et al., 2013; Silva, 2007; Smith, Guller, \& Zapolski, 2013; Stautz \& Cooper, 2013; Tackett, 2006).

However, few studies have examined this relationship in adolescents who are attempting to manage the expectations of 2 different cultures. Zhang, Mandl, \& Wang (2010) assessed the relationship of five personality traits to depression and self-esteem of Chinese college students in Germany. They reported that the traits of neuroticism and conscientiousness were associated with higher levels of depression, while extraversion and openness to experience were related to higher self-esteem. Although not focused on mental health outcomes, a study of Mexican American college students found that neuroticism significantly predicted higher levels of acculturative stress (Mangold, Veraza, Kinkler, \& Kinney, 2007). This finding by Mangold and colleagues suggests that certain traits may influence acculturation differently than other traits and potentially moderate the effect of acculturation on mental health. One study of Asian American college students found that a greater alignment with values of the Western culture was positively correlated with extraversion, conscientiousness, and openness to experience, while acculturation was negatively correlated with neuroticism (Eap et al., 2008). Identical associations between personality traits and acculturation were reported in a study of Chinese undergraduate students in Canada (Ryder, Alden, \& Paulhus, 2000).

Whether personality traits might moderate the risk for mental health problems associated with acculturation is 
still unknown. However, meta-analyses indicate that extraversion, conscientiousness, and openness are associated with more adaptive coping styles when individuals are experiencing distress/adversity (e.g. acculturative stress), and that the interaction between personality and coping style can influence mental health outcomes (Carver \& Connor-Smith, 2010). A study of Korean Americans showed that personality traits do interact with acculturation to affect coping in different ways (Roesch, Wee, \& Vaughn, 2006). In that research, neuroticism was linked to avoidant coping while extraversion, conscientiousness and openness were related to strategies such as positive reframing and acceptance.

\section{Research Aims}

Previous research provides a foundation for understanding the roles that acculturation and personality traits may play in development of mental health problems among Korean American youth. But many questions remain. The purpose of this study was to expand existing knowledge by examining the influence of acculturation, personality traits, and their interaction on the mental health problems of Korean American adolescents. Our specific aims were: 1 ) to determine whether the degree of acculturation and/or specific personality traits were associated with mental health problems of Korean American adolescents; and 2) to determine whether any of these traits would moderate the association between acculturation and mental health problems of the youth.

\section{Methods}

\subsection{Participants and Settings}

The sample included Korean American adolescents who were recruited from Korean churches, Korean language schools, Korean shopping malls and restaurants, mental health centers serving Asian and Pacific Islander communities, and websites for the Korean community in California. Inclusion criteria were: 1) 10 - 19 years old; 2) self-identified as Korean American; and 3) able to communicate in Korean or in English. Adolescents and/or families who could not understand the purpose of the study or the informed consent were excluded.

\subsection{Procedure}

The study was approved by the Committee on Human Research of the University of California, San Francisco. If an adolescent expressed interest in participating, a packet of materials was provided that included self-report measures and an informed written consent for both the adolescent and the parent. Participants returned completed materials by mail or in-person. Follow-up phone calls or e-mails occurred when necessary to gather missing information. If the participant received scores for their mental health problems indicating a level of clinical concern, the researcher discussed the findings with the parent and adolescent and gave them a list of mental health service resources.

\subsection{Measures}

\subsubsection{Demographic Questionnaire}

A 6-item demographic questionnaire was used. The items consisted of gender, age, father and mother's educational level, adequacy of family income, and length of residence in the US.

\subsubsection{Stephenson Multigroup Acculturation Scale}

The Stephenson Multigroup Acculturation Scale (SMAS) is a self-report, 4-point Likert scale to measure one's degree of immersion in dominant American culture versus one's traditional ethnic culture (Stephenson, 2000). Items assess language use, interactions with others, food preferences, and choice of media, reflecting knowledge, behaviors, and attitudes about the 2 cultures (Stephenson, 2000). The Dominant Society Immersion (DSI) subscale of the SMAS was used as the measure of acculturation in this study. One item was deleted because it was not relevant for adolescents to complete. In addition, the words "newspaper" and "Anglo American" were replaced with more current terminology, specifically "magazines/newspaper/non-school related books (online or offline)" and "European American.” The original author reported a Cronbach's alpha score of .86 for the total score with a sample of 436 participants from five ethnic groups, including 8\% Asian Americans. The Cronbach's alpha score for the revised 14-item DSI subscale with this sample of Korean American adolescents 
was .77. Convergent, discriminant validity, and the factor structure of the SMAS have been established (Stephenson, 2000).

\subsubsection{NEO Five-Factor Inventory-3}

The NEO Five-Factor Inventory-3 (NEO-FFI-3) is a self-report measure, uses 4-point Likert scales to measure the 5 personality traits discussed earlier: Neuroticism (N), Extraversion (E), Openness to Experience (O), Agreeableness (A) and Conscientiousness (C) (McCrae \& Costa, 2007). The subscale for each trait contains 12-items. The factor/trait structure was established originally with a sample of 500 adolescents, including about 8\% Asian Pacific Islander adolescents (McCrae \& Costa, 2007). All five factor/trait scores were used in this study. In the original testing of the measure, Cronbach's alpha scores for the subscales ranged from .72 for Agreeableness to .83 for Conscientiousness. Subscale alphas for our sample of adolescents were .80 for Neuroticism, .75 for Extraversion, .75 for Openness, .72 for Agreeableness, and .81 for Conscientiousness.

\subsubsection{Youth Self Report}

The Youth Self Report (YSR) was our measure of mental health problems. It is a questionnaire developed for youth 11 - 18 years of age to measure their problematic feelings, thoughts, and behaviors during the past 6 months (Achenbach \& Rescorla, 2001). The YSR contains 112 items, each on a 3-point Likert scale. The higher the youth's score on the scales, the greater the psychological distress the youth is experiencing. The YSR has eight empirically-based syndrome scales: Anxious/Depressed, Withdrawn/Depressed, Somatic Complaints, Social Problems, Thought Problems, Attention Problems, Rule-Breaking Behavior, and Aggressive Behavior. These are then calculated into composite scores for Internalizing Problems, Externalizing Problems, and Total Problems. The Total Problems score was used for our analyses.

Studies have shown excellent Cronbach's alpha scores for the Total Problems scale ( $\alpha=.93-.95)$ (Achenbach \& Rescorla, 2001; Ebesutani, Bernstein, Martinez, Chorpita, \& Weisz, 2011; Ivanova et al., 2007; Rescorla et al., 2007). The Cronbach's alpha score for the Total Problems scale with this sample of Korean American adolescents was .94. Content validity, criterion-related validity, and concurrent validity of the YSR have been established (Achenbach \& Rescorla, 2001; Ebesutani et al., 2011; van Lang, Ferdinand, Oldehinkel, Ormel, \& Verhulst, 2005). The YSR has been widely tested in Far East Asian countries such as China, Japan, and Korea as well as in Western countries, with excellent reliabilities (Ivanova et al., 2007; Rescorla et al., 2007).

\subsection{Data Analysis}

Descriptive statistics were used to examine sample characteristics, including frequency and percent for gender, father and mother's educational level, and adequacy of family income. Means and standard deviations (SDs) were computed for age and length of residence in the US. Hierarchical linear regression analyses were used to examine the aims. Before examining the aims, assumptions for use of linear regression were tested. In addition, preliminary relationships were computed between the adolescents' mental health problems and all demographic and predictor variables. Pearson's correlation coefficients were used for continuous variables and t-tests for categorical variables. Categorical variables were created for family income and educational level of parents because of their distribution patterns.

To examine Aim 1, demographic variables showing a significant relationship to mental health problems were included in the regression analysis at the first step to control for their effects. In the second step, scores for acculturation and for all personality traits were entered. To examine Aim 2, five separate hierarchical regressions were computed to examine the unique contribution of each personality trait and its interaction to acculturation. In these models, significant demographic variables were entered at the first step, scores for acculturation and one of the personality traits were entered at the second step, and the interaction between acculturation and the trait was entered at the third step. Significance level was set at an alpha level of .05 in all analyses. SPSS version 22 was used to compute statistical procedures.

\section{Results}

The sample included 138 adolescents. 57 participants (41\%) were males and 81 were females (59\%). The mean age of the participants was 14.43 years $(10-19$ years, $S D=2.09)$. Their length of residence in the US ranged from less than 1 to 18 years, with a mean score of 12.36 years $(S D=4.14)$. Approximately $71 \%$ of fathers and 
$66 \%$ of mothers had completed either college or graduate studies. About $61 \%$ of the participants reported that "their family had enough money but no extra", and 36\% reported that "they had more money than they need".

Means and correlations for study variables with continuous scores are shown in Table 1. Length of residence in the US was the only demographic variable that was significantly correlated with mental health problems. Two personality traits were correlated with mental health problems: neuroticism had a high positive correlation while agreeableness had a negative moderate correlation. No significant correlations with mental health problems were found for age, acculturation or the personality traits of extraversion, openness, and conscientiousness. In addition, preliminary t-tests indicated no significant differences in mental health problems for gender, father and mother's educational level, or family income. Based on these preliminary analyses, length of residence was the only demographic variable included in regression analyses for the aims.

Table 2 presents findings for Aim 1. Although length of residence in the US was correlated with mental health in the bivariate analysis, it was not a significant predictor when scores for acculturation and all personality traits were entered in the second step. Consistent with preliminary bivariate correlations, only the 2 personality traits of neuroticism and agreeableness were significant predictors of mental health problems for the adolescents. Neuroticism $(\beta=.63, p<.001)$ was positively associated with mental health problems, while agreeableness $(\beta=-.18, p<.01)$ was negatively associated with mental health problems. The total model explained $52.6 \%$ of the variance in mental health problems $(F(7,115)=18.24, p<.001)$.

Table 1. Means and correlations for age, length of time in the US, acculturation, personality traits, and total mental health problems.

\begin{tabular}{|c|c|c|c|c|c|c|c|c|c|c|}
\hline Variable & $M(S D)$ & 1 & 2 & 3 & 4 & 5 & 6 & 7 & 8 & 9 \\
\hline Age & $14.43(2.09)$ & - & $.50^{* *}$ & .17 & $.19^{*}$ & -.11 & .11 & -.14 & .05 & .14 \\
\hline Time in the US & $12.36(4.14)$ & & - & $.54^{* *}$ & $.19^{*}$ & .02 & .07 & .10 & .04 & $.19^{*}$ \\
\hline Acculturation & $46.22(5.50)$ & & & - & .02 & .17 & $.20^{*}$ & .14 & $.17^{*}$ & .02 \\
\hline Neuroticism & $25.62(7.52)$ & & & & - & -.09 & .12 & $-.21^{*}$ & -.16 & $.68^{* *}$ \\
\hline Extraversion & 30.91 (5.88) & & & & & - & $.19^{*}$ & .07 & .15 & -.08 \\
\hline Openness & $28.53(6.37)$ & & & & & & - & .14 & .04 & .14 \\
\hline Agreeableness & 31.55 (5.91) & & & & & & & - & .17 & $-.30^{* *}$ \\
\hline Conscientiousness & 29.10 (6.49) & & & & & & & & - & -.12 \\
\hline Total problems & 45.30 (23.65) & & & & & & & & & - \\
\hline
\end{tabular}

Table 2. Effects of time in the US, acculturation, and personality traits on mental health problems.

\begin{tabular}{cccc}
\hline Variable & B & SE B & $\beta$ \\
\hline Step 1 & & & $.20^{*}$ \\
Time in the US & 1.18 & .52 & .13 \\
Step 2 & & .45 & -.04 \\
Time in the US & .74 & .36 & $.63^{* * *}$ \\
Acculturation & -.20 & .22 & -.04 \\
Neuroticism & 1.96 & .27 & .11 \\
Extraversion & -.15 & .26 & $-.18^{* *}$ \\
Openness & .43 & .27 & .04 \\
Agreeableness & -.74 & .25 & \\
\hline
\end{tabular}

$R^{2}=.04$ for Step $1 ; R^{2}$ change $=.48$ for Step $2(p<.001) .{ }^{*} p<.05,{ }^{* *} p<.01,{ }^{* * *} p<.001$. 
In the 5 separate regression models that were computed for each personality trait, only 1 trait showed a significant interaction with acculturation: the interaction between acculturation and the trait of openness. Table 3 shows the findings for this regression. In the final model (Step 3), length of residence in the US had a significant relationship to mental health problems ( $\beta=.29, p<.01$ ), explaining $4.2 \%$ of the variance. Acculturation was not a significant predictor when its direct effects were examined, although it did show a trend toward significance ( $\beta$ $=.85, p=.054)$. The direct effect of the openness trait was significant $(\beta=2.18, p<.05)$. Together, acculturation and openness explained an additional 3.4\% of the variance in mental health problems ( $F$ change $=2.21, p$ $=.11$ ). Finally, the interaction between acculturation and openness accounted for an additional $4 \%$ of the variance in mental health problems ( $F$ change $=5.63, \beta=-2.42, p<.05$ ). To better understand the interaction effect, correlations were computed between acculturation and mental health problems for adolescents with low versus high openness to experience. Youth were split at the mean for openness to determine these groups and partial correlations were computed that controlled for length of residence. Correlations indicated that for youth who were high in openness to experience, greater acculturation was associated with fewer mental health problems $(r=-.25, p<.05)$. However, for youth who were low in openness to experience, there was no relationship at all between acculturation and mental health problems $(r=.004, p=.97)$. The overall regression model was significant, with all variables accounting for $12 \%$ of the variance in mental health problems for Korean American adolescents in the sample $(F(4,120)=3.97, p<.01)$.

\section{Discussion}

This study examined the relationship of acculturation and personality traits to mental health problems of Korean American adolescents as well as how personality might moderate any effect of acculturation on their mental health problems. Findings related to Aim 1 indicate that acculturation played a minimal role in predicting mental health problems of these youth, although longer residence in the US was predictive of more problems. However, 2 personality traits were very strong predictors. When adolescents had greater "neuroticism" (i.e. stress reactivity and emotional instability), they were at significantly higher risk for mental health problems. In contrast, adolescents whose dispositions were more "agreeable" (i.e. altruistic and cooperative) were less likely to have mental health problems. Personality traits did not appear to have any major moderating effect overall, with the exception of "openness to experience" (Aim 2). Greater acculturation appeared to serve as a protective factor for youth who were more open to experience (i.e. curious and independent in their judgments) but not for youth who were less open to experience.

\subsection{Acculturation and Mental Health}

Our results for acculturation differ from those of Yeh (2003) who found that greater acculturation was associated with fewer mental health problems for Korean American youth. However, findings reported by

Table 3. Effects of acculturation, openness to experience, and their interaction on mental health problems.

\begin{tabular}{cccc}
\hline Variable & $B$ & $S E B$ & $\beta$ \\
\hline Step 1 & & & $.20^{*}$ \\
Time in the US & 1.18 & .51 & $.27^{* *}$ \\
Step 2 & & & -.16 \\
Time in the US & 1.58 & .60 & .15 \\
Acculturation & -.71 & .47 & .34 \\
Openness to experience & .58 & & $.29^{* *}$ \\
Step 3 & 1.67 & .59 & .85 \\
Time in the US & 3.84 & 1.97 & $2.18^{*}$ \\
Acculturation & 8.38 & 3.31 & $-2.42^{*}$ \\
Openness to experience & -.16 & .07 & \\
Acculturation x openness
\end{tabular}

$R^{2}=.04$ for Step $1 ; R^{2}$ change $=.03$ for Step $2(p=.11) ; R^{2}$ change $=.04$ for Step $3(p<.05) .{ }^{*} p<.05,{ }^{* *} p<.01$. 
Cho \& Bae (2005) are consistent with our results that acculturation is not a significant predictor of mental health problems for Korean American youth. Our results extend the findings of Cho \& Bae (who studied internalizing problems only) to all types of mental health problems. It is important to note that we controlled for length of residence before accounting for any acculturation effect. Length of residence was strongly associated with acculturation in our preliminary analysis so it is possible that length of residence measured aspects of acculturation that were predicting mental health problems. Still, this would not explain why our preliminary bivariate correlations showed a significant relationship between length of residence and mental health but no significant relationship between acculturation and mental health. It will be important to better understand how length of residence per se may affect mental health problems differently than specific changes in language, attitudes or behaviors that are reflected in acculturation level.

\subsection{Neuroticism and Mental Health}

Our finding regarding neuroticism is consistent with results of a previous study for Chinese adolescent immigrants, showing a significant relationship between neuroticism and depression (Zhang et al., 2010). Neuroticism is described as a trait of being reactive to and more easily distressed by stressors in the environment rather than feeling secure or confident in managing environmental demands (McCrae \& Costa, 2007). Individuals who are higher on neuroticism tend to experience unpleasant emotions such as anger, anxiety or depression more easily, reflecting greater difficulty in regulating their emotions. Mangold and colleagues (2007) found that neuroticism was associated with higher levels of acculturative stress among Mexican immigrants. Adolescents in our sample who were more easily distressed and susceptible to negative emotions may also have experienced greater acculturative stress, increasing their vulnerability to mental health problems.

\subsection{Agreeableness and Mental Health}

Our finding regarding the link between agreeableness and fewer mental health problems has not been reported previously among immigrant youth. However, there is a body of literature indicating that agreeableness is associated with subjective well-being (Haslam, Whelan, \& Bastian, 2009), greater psychological resilience in adolescents (Fayombo, 2010), and better mental health outcomes among college students (Shirazi, Khan, \& Ansari, 2012). A longitudinal study found that 8 year olds who were high on agreeableness had fewer behavior problems and had lower levels of depression and substance abuse 25 years later (Laursen, Pulkkinen, \& Adams, 2002). The trait of agreeableness has been described as the ability to get along well with others, emphasizing cooperation, trustfulness, altruism, compassion, empathy, and control of one's negative emotions during interactions (McCrae \& Costa, 2007; Ryckman, 2004). People may respond to adolescents who have these characteristics more positively, regardless of their alignment with Korean or American values. Greater social acceptance may then create a more supportive environment that contributes to better mental health for these Korean youth.

\subsection{The Moderating Effect of Openness to Experience}

The moderating effect of openness to experience was one of our most interesting findings. To our knowledge, we are the first study to report this result. As noted earlier, there was a negative relationship between acculturation and mental health problems for adolescents who were higher on the trait of openness to experience but there was no relationship between acculturation and mental health among adolescents who were lower on openness. Individuals who are more open to experience are described as having greater curiosity about and appreciation for new and diverse experiences rather than traditional interests and familiar activities (McCrae \& Costa, 2007). Thus, they would likely have a greater interest in exploring and learning about new cultures and be less influenced by others who espouse more traditional values. For these adolescents, their greater alignment with the American culture was a protective factor for their mental health. However, less acculturation among these youth was associated with more mental health problems. Because of the high value they place on novelty and continued growth, those who do not feel more fully acculturated may experience greater acculturative and emotional distress and a resulting risk for mental health problems.

\subsection{Implications for Research and Practice}

Results of this study indicate that length of residence in the US was associated with both acculturation and 
greater mental problems for Korean American adolescents. Length of residence is likely linked to generation status so future research should more specifically examine its potential moderating role. Most Korean American adolescents are second or higher generation; only about 30\% are first generation (Cho \& Bae, 2005; Kim \& Cain, 2008; Yeh, 2003). It will be important to understand why adolescents who are first generation may have better mental health and to identify factors that enhance their resilience.

In addition, there is a need to examine how acculturation and personality traits are related to specific mental health problems, with attention to whether they have a greater impact on internalizing problems such as anxiety and depression or externalizing problems such as rule-breaking behavior and aggression. Acculturation may influence some problems more than others. Similarly, research is essential to identify which mental health problems are most affected by neuroticism or lack of agreeableness and the underlying mechanisms responsible for their effects. Research with a larger sample size will also be required to more carefully examine the moderating effects of openness to experience. A larger sample size will help to detect other potential moderating effects as well. Lastly, it will be important to examine whether personality traits identified in this research influence mental health similarly across cultures and age groups or whether they are unique to Korean American youth.

Findings have implications for identification of youth at greater risk of mental health problems and for development of prevention programs targeted to adolescents with specific personality traits. Because neuroticism and less agreeableness may result in greater vulnerability to mental health problems, assessment of these risk factors should be considered as part of mental health evaluations of Korean American adolescents. For example, some brief questions about their personality traits can be asked by pediatric and adolescent clinicians during well-adolescent visits. In addition, interventions may be offered to provide additional support such as individual or group counseling, education, and family intervention for adolescents with these traits or to help them consider other options for how they might view and relate to others. Considering many Korean American adolescents go to Korean language schools or churches, these sites may offer good environments to deliver these interventions.

\subsection{Limitations}

Although this study enhances knowledge of factors that may influence mental health of Korean American youth, it has limitations. One limitation is its cross-sectional design that does not allow for interpretation of causality. It is impossible to determine whether acculturation and personality traits of neuroticism and less agreeableness increase mental health problems, or whether mental health problems exacerbate or trigger existing or latent predispositions. Longitudinal studies are needed to determine whether personality traits measured early in life predict the development of later mental health problems or interact with acculturation differently over time.

Another limitation is the use of a convenience sample from only one state in the western US. Findings may not be generalizable to the larger Korean American population in the US. Moreover, the convenience sample might not exhibit a representative distribution of mental health problems. A larger, probability-based sample from multiple sites nationwide is recommended for future research.

Lastly, all measures were self-report. While self-report is essential for understanding internally-based emotions and attitudes, its accuracy relies on the reliability of respondents' perceptions and recall. The use of a structured interview by a mental health professional would strengthen assessment of adolescent mental health problems in future research. The addition of parent report could also contribute to a more comprehensive assessment of adolescent behavior.

\section{References}

Achenbach, T. M., \& Rescorla, L. A. (2001). Manual for the ASEBA School-Age Forms and Profiles. Burlington, VT: University of Vermont Research Center for Children, Youth, \& Families.

Carver, C. S., \& Connor-Smith, J. (2010). Personality and Coping. Annual Review of Psychology, 61, 679-704. http://dx.doi.org/10.1146/annurev.psych.093008.100352

Cho, S., \& Bae, S.-W. (2005). Demography, Psychosocial Factors, and Emotional Problems of Korean American Adolescents. Adolescence, 40, 533-550.

Cho, S.-J., Hudley, C., \& Back, H. J. (2003). Cultural Influences on Ratings of Self-Perceived Social, Emotional, and Academic Adjustment for Korean American Adolescents. Assessment for Effective Intervention, 29, 3-14. http://dx.doi.org/10.1177/073724770302900102

Cyders, M. A., Flory, K., Rainer, S., \& Smith, G. T. (2009). The Role of Personality Dispositions to Risky Behavior in Pre- 
dicting First-Year College Drinking. Addiction, 104, 193-202. http://dx.doi.org/10.1111/j.1360-0443.2008.02434.x

Eap, S., DeGarmo, D. S., Kawakami, A., Hara, S. N., Hall, G. C. N., \& Teten, A. L. (2008). Culture and Personality among European American and Asian American Men. Journal of Cross-Cultural Psychology, 39, 630-643. http://dx.doi.org/10.1177/0022022108321310

Ebesutani, C., Bernstein, A., Martinez, J. I., Chorpita, B. F., \& Weisz, J. R. (2011). The Youth Self Report: Applicability and Validity across Younger and Older Youths. Journal of Clinical Child and Adolescent Psychology, 40, 338-346. http://dx.doi.org/10.1080/15374416.2011.546041

Fayombo, G. (2010). The Relationship between Personality Traits and Psychological Resilience among Caribbean Adolescents. International Journal of Psychological Studies, 2, 105-116. http://dx.doi.org/10.5539/ijps.v2n2p105

Haslam, N., Whelan, J., \& Bastian, B. (2009). Big Five Traits Mediate Associations between Values and Subjective Well-Being. Personality and Individual Differences, 46, 40-42. http://dx.doi.org/10.1016/j.paid.2008.09.001

Hopwood, C. J., \& Grilo, C. M. (2010). Internalizing and Externalizing Personality Dimensions and Clinical Problems in Adolescents. Child Psychiatry and Human Development, 41, 398-408. http://dx.doi.org/10.1007/s10578-010-0175-4

Ivanova, M. Y., Achenbach, T. M., Dumenci, L., Rescorla, L. A., Almqvist, F., Weintraub, S. et al. (2007). Testing the 8-Syndrome Structure of the Child Behavior Checklist in 30 Societies. Journal of Clinical Child and Adolescent Psychology, 36, 405-417. http://dx.doi.org/10.1080/15374410701444363

Kim, E., \& Cain, K. C. (2008). Korean American Adolescent Depression and Parenting. Journal of Child and Adolescent Psychiatric Nursing, 21, 105-115. http://dx.doi.org/10.1111/j.1744-6171.2008.00137.x

Krueger, R. F. (1999). Personality Traits in Late Adolescence Predict Mental Disorders in Early Adulthood: A Prospective-Epidemiological Study. Journal of Personality, 67, 39-65. http://dx.doi.org/10.1111/1467-6494.00047

Krueger, R. F., \& Eaton, N. R. (2010). Personality Traits and the Classification of Mental Disorders: Toward a More Complete Integration in DSM-5 and an Empirical Model of Psychopathology. Personality Disorders: Theory, Research and Treatment, 1, 97-118. http://dx.doi.org/10.1037/a0018990

Krueger, R. F., Caspi, A., Moffitt, T. E., Silva, P. A., \& McGee, R. (1996). Personality Traits Are Differentially Linked to Mental Disorders: A Multitrait-Multidiagnosis Study of an Adolescent Birth Cohort. Journal of Abnormal Psychology, 105, 299-312. http://dx.doi.org/10.1037/0021-843X.105.3.299

Laursen, B., Pulkkinen, L., \& Adams, R. (2002). The Antecedents and Correlates of Agreeableness in Adulthood. Developmental Psychology, 38, 591-603. http://dx.doi.org/10.1037/0012-1649.38.4.591

Lee, G., Yim, K., Curry, T., \& Rodriguez, S. F. (2012). The Relevance of Social and Cultural Contexts: Religiosity, Acculturation and Delinquency among Korean Catholic Adolescents in Southern California. The Social Science Journal, 49, 537-544. http://dx.doi.org/10.1016/j.soscij.2012.06.001

Malouff, J. M., Thorsteinsson, E. B., \& Schutte, N. S. (2005). The Relationship between the Five-Factor Model of Personality and Symptoms of Clinical Disorders: A Meta-Analysis. Journal of Psychopathology and Behavioral Assessment, 27, 101-114. http://dx.doi.org/10.1007/s10862-005-5384-y

Mangold, D. L., Veraza, R., Kinkler, L., \& Kinney, N. A. (2007). Neuroticism Predicts Acculturative Stress in Mexican American College Students. Hispanic Journal of Behavioral Sciences, 29, 366-382. http://dx.doi.org/10.1177/0739986307302167

McCrae, R. R., \& Costa Jr., P. T. (2007). Brief Versions of the NEO-PI-3. Journal of Individual Differences, 28, 116-128. http://dx.doi.org/10.1027/1614-0001.28.3.116

McCrae, R. R., \& Costa Jr., P. T. (2008). The Five-Factor Theory of Personality. In O. P. John, R. W. Robins, \& L. A. Pervin (Eds.), Handbook of Personality: Theory and Research (3rd ed., pp. 159-181). New York: Guilford Press.

Moore, M. N., Salk, R. H., Van Hulle, C. A., Abramson, L. Y., Hyde, J. S., Lemery-Chalfant, K., \& Goldsmith, H. H. (2013). Genetic and Environmental Influencs on Rumination, Distraction, and Depressed Mood in Adolescence. Clinical Psychological Science, 1, 316-322. http://dx.doi.org/10.1177/2167702612472884

Rescorla, L. A., Achenbach, T. M., Ivanova, M. Y., Dumenci, L., Almqvist, F., Bilenberg, N., Verhulst, F. et al. (2007). Behavioral and Emotional Problems Reported by Parents of Children Ages 6 to 16 in 31 Societies. Journal of Emotional and Behavioral Disorders, 15, 130-142. http://dx.doi.org/10.1177/10634266070150030101

Roesch, S. C., Wee, C., \& Vaughn, A. A. (2006). Relations between the Big Five Personality Traits and Dispositional Coping in Korean Americans: Acculturation as a Moderating Factor. International Journal of Psychology, 41, 85-96. http://dx.doi.org/10.1080/00207590544000112

Ryckman, R. M. (2004). Theories of Personality (8th ed.). Belmont, CA: Thomas/Wadsworth.

Ryder, A. G., Alden, L. E., \& Paulhus, D. L. (2000). Is Acculturation Unidimensional or Bidimensional? A Head-to-Head Comparison in the Prediction of Personality, Self-Identity, and Adjustment. Journal of Personality and Social Psychology, 79, 49-65. http://dx.doi.org/10.1037/0022-3514.79.1.49 
Shirazi, M., Khan, M. A., \& Ansari, F. A. (2012). Mental Health in Relation to Personality Characteristics among Professional and Nonprofessional Students. Journal of Arts, Science and Commerce, 3, 8-15.

Silva, A. S. (2007). The Relationship between Personality Traits and Eating Pathology in Adolescent Girls. Archives of Women's Mental Health, 10, 285-292. http://dx.doi.org/10.1007/s00737-007-0199-3

Smith, G. T., Guller, L., \& Zapolski, T. C. (2013). A Comparison of Two Models of Urgency: Urgency Predicts Both Rash Action and Depression in Youth. Clinical Psychological Science, 1, 266-275. http://dx.doi.org/10.1177/2167702612470647

Stautz, K., \& Cooper, A. (2013). Impulsivity-Related Personality Traits and Adolescent Alcohol Use: A Meta-Analytic Review. Clinical Psychology Review, 33, 574-592. http://dx.doi.org/10.1016/j.cpr.2013.03.003

Stephenson, M. (2000). Development and Validation of the Stephenson Multigroup Acculturation Scale (SMAS). Psychological Assessment, 12, 77-88. http://dx.doi.org/10.1037/1040-3590.12.1.77

Tackett, J. L. (2006). Evaluating Models of the Personality-Psychopathology Relationship in Children and Adolescents. Clinical Psychology Review, 26, 584-599. http://dx.doi.org/10.1016/j.cpr.2006.04.003

United States Census Bureau (2012). Census Briefs. The Asian Population: 2010. Washinton DC: US Department of Commerce.

Van Lang, N. D. J., Ferdinand, R. F., Oldehinkel, A. J., Ormel, J., \& Verhulst, F. C. (2005). Concurrent Validity of the DSM-IV Scales Affective Problems and Anxiety Problems of the Youth Self-Report. Behaviour Research and Therapy, 43, 1485-1494. http://dx.doi.org/10.1016/j.brat.2004.11.005

Widiger, T. A., \& Costa Jr., P. T. (2012). Integrating Normal and Abnormal Personality Structure: The Five-Factor Model. Journal of Personality, 80, 1471-1506. http://dx.doi.org/10.1111/j.1467-6494.2012.00776.x

Yeh, C. J. (2003). Age, Acculturation, Cultural Adjustment, and Mental Health Symptoms of Chinese, Korean, and Japanese Immigrant Youths. Cultural Diversity and Ethnic Minority Psychology, 9, 34-48. http://dx.doi.org/10.1037/1099-9809.9.1.34

Zhang, J., Mandl, H., \& Wang, E. (2010). Personality, Acculturation, and Psychosocial Adjustment of Chinese International Students in Germany. Psychological Reports, 107, 511-525. http://dx.doi.org/10.2466/07.09.11.17.PR0.107.5.511-525

Submit or recommend next manuscript to SCIRP and we will provide best service for you:

Accepting pre-submission inquiries through Email, Facebook, LinkedIn, Twitter, etc.

A wide selection of journals (inclusive of 9 subjects, more than 200 journals)

Providing 24-hour high-quality service

User-friendly online submission system

Fair and swift peer-review system

Efficient typesetting and proofreading procedure

Display of the result of downloads and visits, as well as the number of cited articles

Maximum dissemination of your research work

Submit your manuscript at: http:/papersubmission.scirp.org/ 